\title{
Plasma and tissue proteomic prognostic factors of response in primary breast cancer patients receiving neoadjuvant chemotherapy
}

\author{
J. BONNETERRE ${ }^{1,2}$, F. RÉVILLION ${ }^{1}$, C. DESAUW ${ }^{3}$, E. BLOT ${ }^{4}$, A. KRAMAR ${ }^{1}$, \\ C. FOURNIER ${ }^{1}$, L. HORNEZ ${ }^{1}$ and J.P. PEYRAT ${ }^{1}$ \\ ${ }^{1}$ Oscar Lambret Center, F-59000 Lille; ${ }^{2}$ Lille-North of France University, F-59000 Lille; \\ ${ }^{3}$ Regional University Hospital, F-59037 Lille Cedex; ${ }^{4}$ Henri Becquerel Center, F-76038 Rouen Cedex, France
}

Received July 16, 2012; Accepted August 30, 2012

DOI: 10.3892/or.2012.2090

\begin{abstract}
A pathological complete response (pCR) after neoadjuvant chemotherapy is observed in approximately $20 \%$ of breast cancer patients. A proteomic analysis was performed on plasma and tumor tissue before treatment to evaluate its potential impact on the prediction of response. One hundred and forty-nine breast cancer patients eligible for neoadjuvant chemotherapy were included in the study between February 2004 and January 2009 at three centers. The proteomic analysis was performed using SELDI Technology (ProteinChip CM10 pH4, IMAC-Cu and H50). Three acquisition protocols were used according to the mass range. Plasma and tumor proteomic signatures were generated using generalized ROC criteria and cross-validation. Twenty-eight (18.8\%) patients out of 149 experienced a pCR according to Sataloff criteria. In the cytosol analysis, respectively 4,2 and 8 proteins had significantly different levels of expression in the responders and non-responders using IMAC-Cu, H50 and CM10 pH4. Among the 8 proteins of interest on $\mathrm{CM} 10 \mathrm{pH} 4,2$ (C1 and C7) were selected and were validated in 95.0 and $85.6 \%$ of the models. In the plasma analysis, respectively 12,6 and 2 proteins had different levels of expression using the same ProteinChips. Among the 12 plasma proteins of interest on IMAC-Cu, 2 (P1 and P7) were selected and were validated in 94.8 and $97.6 \%$ of the models. A combined proteomic signature was generated, which remained statistically significant when adjusted for hormone receptor status and Ki-67. Our results show that proteomic analysis can differentiate complete pathological responders in breast cancer patients after neoadjuvant chemotherapy.
\end{abstract}

Correspondence to: Professor Jacques Bonneterre, Oscar Lambret Center, 3 rue Frédéric Combemale, F-59020 Lille, France

E-mail: j-bonneterre@o-lambret.fr

Key words: neoadjuvant chemotherapy, breast cancer, proteomic analysis

\section{Introduction}

Breast cancer is currently the most common female cancer. There are 183,000 new cases each year and 41,000 women succumb to this disease in the USA in 2000 (1). In $85 \%$ of cases the tumor remains localized and the treatment consists of surgery, radiotherapy \pm chemo and/or hormonotherapy. Despite this treatment, local or distant relapses occur in approximately $40 \%$ of patients. Predictive risk factors are being used to a greater extent to identify subgroups of patients who will most likely benefit from adjuvant treatment. The use of the St. Gallen guidelines (2) has led to the treatment of approximately $50 \%$ of patients after surgery for early breast cancer with anthracyclin-based chemotherapy sequenced with taxanes (3). This probabilistic criterion has resulted in many patients being submitted to aggressive treatments.

Neoadjuvant chemotherapy is widely used in the management of patients with locally advanced breast cancer. In addition to increasing the rates of breast conservation, this treatment strategy allows the use of pathologic response as an early surrogate marker for overall survival. The uncertain benefit, the toxicity of chemotherapy and the existence of alternative treatments calls for the development of methods to select patients most likely to benefit.

Using conventional two-dimensional electrophoresis and mass spectrometry, we previously identified markers of potential clinical interest in human breast cancer, such as

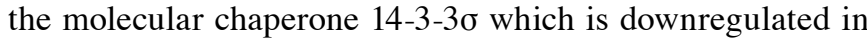
breast cancer cells as compared with normal breast epithelial cells (4). Surface-enhanced laser desorption/ionization-time of flight (SELDI-TOF) mass spectrometry (MS) coupled with appropriate bio-informatic tools have been used to identify protein patterns related to various stages and types of solid tumors and serum (5-12). This technology combines chromatographic fractionation of the proteome using protein biochips and TOF MS analysis that can be applied to various clinical samples, such as serum and tissue (13); it allows relatively high-throughput protein analysis of complex biological samples, with limited preprocessing steps.

To date, most proteomic-based studies are largely performed in vitro for the identification of differential expression levels between parental and chemotherapy-resistant cell 
sublines. Only a few small studies are based on fresh breast cancer tissue samples $(14,15)$ and plasma proteomic evaluations in this situation have not yet been published.

Herein, we define plasma and tumor proteomic profiles of primary breast cancer patients and provide evidence that such an approach may have a significant impact in predicting response to neoadjuvant chemotherapy. The primary objective of this study was to define proteomic signatures correlated with a complete pathological response after neoadjuvant chemotherapy. Secondary objectives included the study of correlations between proteomic signatures and nodal involvement, pathological subtypes, clinical and ultrasound response and menopausal status.

\section{Materials and methods}

Patients. Main inclusion criteria were: female patients over 18 years of age, with a histologically proven breast adenocarcinoma, eligible for neoadjuvant chemotherapy with anthracyclines and taxanes with no previous chemotherapy for malignant disease. Information and informed consent were obtained according to the French law. Exclusion criteria were metastatic disease, non-adenocarcinoma breast tumors, contraindication of anthracyclines/taxanes, patient's refusal of storage of blood and tumor biopsy and inclusion in another clinical research study.

Treatment. The chemotherapy protocol included a sequence of FEC100 (epirubicin, $100 \mathrm{mg} / \mathrm{m}^{2}$; cyclophosphamide, $500 \mathrm{mg} / \mathrm{m}^{2}$ and fluorouracil, $500 \mathrm{mg} / \mathrm{m}^{2}$ ) followed by docetaxel, $100 \mathrm{mg} / \mathrm{m}^{2}$ on Day 1 every 3 weeks, provided the neutrophil cell count was over $1,500 / \mathrm{mm}^{3}$. At the beginning of the study, all patients received 4 cycles of each regimen. After the publication of the results of the PACS 01 adjuvant trial (3), it was decided to administer 3 courses of each for a total of 6 .

Pathological analysis. The pathological diagnosis included the following variables: histological type (essentially ductular or lobular), histoprognostic grading, estradiol and progesterone receptor status, HER2 positivity on immunohistochemistry or FISH, and Ki-67 hyperexpression. In triple-negative tumors (TNBC), the basal phenotype was assessed by determining c-Kit and R EGF hyperexpression.

Inprints of the frozen part of the tumor were performed to be sure that the tissue extract proteomic analysis was performed on tumor tissue. The pathological diagnosis, using the same criteria, was also performed after surgery. The pathological tumor and nodal responses were assessed according to Sataloff (16) criteria.

\section{Protein expression profiling}

Tumor samples. All tumor samples were processed within $1 \mathrm{~h}$ after collection and rapidly frozen at $-80^{\circ} \mathrm{C}$.

Plasma samples. Plasma (10 $\mathrm{ml}$ of blood) was obtained before the initiation of neoadjuvant chemotherapy and processed within $1 \mathrm{~h}$ after collection and frozen at $-80^{\circ} \mathrm{C}$.

Preparation of cytosols. The frozen tissues were weighed, then disrupted and homogenized in $200 \mu \mathrm{l}$ of $50 \mathrm{mM}$ Tris- $\mathrm{HCl}$ buffer ( $\mathrm{pH}$ 9.0) containing $7 \mathrm{M}$ urea, $2 \mathrm{M}$ thiourea, 2\% CHAPS using a Potter Homogenizer and a Rotor-Stator homogenizer (Ribolyser, Hybaid). The homogenate was ultracentrifuged at $105,000 \mathrm{x} \mathrm{g}$ for $60 \mathrm{~min}$ at $4^{\circ} \mathrm{C}$.

The protein concentration of the cytosols was determined using the Quick Start Bradford Dye Reagent (Bio-Rad Laboratories Inc., France) based on the method of Bradford (17).

ProteinChip array analysis. Three types of ProteinChips (Bio-Rad Laboratories) with a surface chemistry of hydrophobic (H50), cationic (CM10) and metal affinity (IMAC-Cu) were tested to determine which might provide the best plasma and tumor cytosol profiles.

Plasma samples were aliquoted $(50 \mu \mathrm{l})$ and thawed at $-80^{\circ} \mathrm{C}$. Each sample $(10 \mu \mathrm{l})$ was denaturated by adding $90 \mu \mathrm{l}$ of $50 \mathrm{mM}$ Tris- $\mathrm{HCl}$ buffer ( $\mathrm{pH} 9.0)$ containing $7 \mathrm{M}$ urea, $2 \mathrm{M}$ thiourea and 2\% CHAPS [(3-cholamidopropyl) dimethylammonio]-propanesulfonic acid). The mixture was vortex-mixed and shaken for $20 \mathrm{~min}$ at room temperature. Tumor cytosols (100 $\mu \mathrm{g}$ proteins) were diluted in $200 \mu \mathrm{l}$ of $50 \mathrm{mM}$ Tris- $\mathrm{HCl}$ buffer $\mathrm{pH} 9.0$ containing $7 \mathrm{M}$ urea, $2 \mathrm{M}$ thiourea and $2 \%$ CHAPS.

The denatured plasma samples $(20 \mu 1)$ and the diluted tumor cytosol samples $(20 \mu \mathrm{l})$ were then diluted (1:10) with the adequate binding buffer (acetonitrile $10 \mathrm{ml} / \mathrm{l}$, trifluoroacetic acid $1 \mathrm{ml} / \mathrm{l}, \mathrm{NaCl} 150 \mathrm{mM}$ for $\mathrm{H} 50$, sodium acetate $100 \mathrm{mM}$ $\mathrm{pH} 4.0$ for CM10 and phosphate-buffer saline (PBS) pH 7.4 for IMAC-Cu). Then $100 \mu 1$ was spotted on the ProteinChip array in a 96-well bioprocessor (Bio-Rad Laboratories). IMAC-Cu ProteinChips were precharged for activation with $50 \mathrm{mM}$ $\mathrm{CuSO}_{4}$ for $10 \mathrm{~min}$ according to the manufacturer's instructions (Bio-Rad Laboratories).

After the samples were allowed to bind at room temperature for $45 \mathrm{~min}$ on a platform shaker (Heidolph Titramax 100), the arrays were washed twice with $200 \mu 1$ adequate binding buffer for $5 \mathrm{~min}$, followed by two quick rinses with $200 \mu \mathrm{l}$ deionized water. After air-drying, $1 \mu \mathrm{l}$ of saturated sinapinic acid [5 mg dissolved in $400 \mu \mathrm{l}$ of acetonitrile/trifluoroacetic acid $(50 \% / 0.5 \%)]$ was applied twice to each spot, allowing the array surface to air-dry 10 min between each application. Proteins bound to the ProteinChips arrays were detected with the ProteinChip System Series 4000 (Bio-Rad Laboratories).

Time of flight spectra were generated by averaging 530 laser shots collected at a laser intensity of 2,500 with a focus mass of 7,000 (for 1,800-10,000 Da proteins), at a laser intensity of 3,500 with a focus mass of 16,000 (for 10,000-20,000 Da proteins), and finally at a laser intensity of 5,000 with a focus mass of 40,000 (for 20,000-150,000 Da proteins). External mass calibration was performed using the All-In-One Peptide molecular mass standard. Spectra analyses (peak detection, mass calibration, baseline substraction and total ion current normalization) were performed using Ciphergen ProteinChip Data Manager DE Software 4.1.

Reproducibility was estimated using one pool of plasma and one pool of tumor cytosols. The mean of the CV, estimated on all the detected peaks both at each laser intensity tested and on each type of ProteinChip array, ranged from 10 to $20 \%$.

Statistical methods. Clinical and echographic assessments of tumor size, node involvement and tissue diagnosis were 
recorded at baseline and at the end of treatment. Patients with a pathological complete response (pCR) were considered responders: tumor $\mathrm{A}(\mathrm{TA}) /$ node $\mathrm{A}(\mathrm{NA})$ or TA/NB. All other categories were considered non-responders.

On the basis of an expected pCR rate of $20 \%$, the inclusion of 100 breast cancer patients treated with neoadjuvant chemotherapy allowed a $95 \%$ confidence interval width of $\pm 8 \%$. Initial demographic and tumor characteristics for categorical variables are presented as frequencies and percentages. Continuous variables are presented as medians and range. For the receiver operating characteristic curve (ROC) analyses, cytosol and plasma variables were transformed to the logarithmic scale if deemed necessary in order to stabilize the variance.

The search for candidate proteins was first performed by univariate analysis using the non-parametric Wilcoxon test comparing responders and non-responders. Multivariate analyses using a generalized ROC criterion were then performed to obtain a proteomic signature separately for cytosol and plasma and then in combination (18). This technique selects the variable combinations which maximize the area under the curve (AUC). Each cytosol and plasma model was then validated internally on 500 random samples with replacement on the whole dataset. Logistic regression models were then applied selecting variables according to the Akaike Information Criterion (AIC). The percentage of times each variable was selected was extracted. Only those variables which were selected in $>80 \%$ of models were retained. Results of statistical tests were considered significant at the $5 \%$ level. The final model was adjusted for significant clinical variables.

\section{Results}

Demographic and clinical characteristics. One hundred and forty-nine breast cancer patients were enrolled between February 2004 and January 2009 from 3 centers. A total of 8 patients were excluded: 4 were ineligible (prior chemotherapy in one and metastatic disease in 3); and 4 were non-evaluable (one patient was untreated, one patient received only one cycle due to toxicity, one had no tumor nor blood plasma sample and one patient developed a carcinomatous meningitis while receiving neoadjuvant chemotherapy).

Clinical characteristics for the 141 patients analyzed are presented in Table I. Median age was 46 years (range, 26-74). Median tumor size was $40 \mathrm{~mm}$ (range, 10-130). All patients received a minimum of three cycles of chemotherapy. Overall, $82(58 \%)$ patients received 3 cycles of FEC and 3 cycles of docetaxel; 38 patients received eight cycles. The reason for not receiving 6 or 8 cycles were side effects which prevented the continuation of the same regimen.

Overall, 89 patients $(63 \%)$ had conservative surgery, and $136(96 \%)$ had an axillary clearance; $98 \%$ of patients had surgery within 6 weeks after the last chemotherapy cycle.

The pathological complete response rate according to Sataloff was $18.8 \%$ (95\% CI, 12.6-25.7). Negative hormone receptor tumors showed a significantly higher pCR rate then positive ones. In addition, in spite of many missing data for Ki-67, not a single patient with Ki-67 $<15 \%$ had a pCR (Table II).

Cytosols. One hundred and eight patients (77\%) were evaluable for cytosol analysis. Using IMAC-Cu and H50 arrays, 150
Table I. Characteristics of the breast cancer patients before chemotherapy.

\begin{tabular}{cc}
\hline Characteristics & $\mathrm{n}=141$ \\
$\mathrm{n}(\%)$
\end{tabular}

$\begin{array}{ll}\text { Site of tumor } & \\ \text { Right } & 76(54) \\ \text { Left } & 65(46)\end{array}$

Tumor stage

T1

$\mathrm{T} 2$

T3

$\mathrm{T} 4$

Nodal involvement

N0

$\mathrm{N} 1$

$\mathrm{N} 2$

Missing

Histology

Ductal

Lobular

Other

Missing

Differentiation

Well

Medium

Poor

SBR grade

I

II

III

NP

17

Missing

15

Hormone receptor status

$\mathrm{ER}^{+} \mathrm{PR}^{+}$

$\mathrm{ER}^{+} \mathrm{PR}^{-}$

$\mathrm{ER}^{-} \mathrm{PR}^{+}$

$\mathrm{ER}^{-} \mathrm{PR}$

Ki-67 index

$<15$

$10 \quad(7)$

$\geq 15$

40 (93)

Missing

91

NP, non-gradable; SBR, Scarff-Bloom-Richardson; ER, estrogen receptor; $\mathrm{PR}$, progesterone receptor.

and 131 different proteins were observed respectively and their levels were statistically different between responders and nonresponders for 4 proteins (IMAC-Cu) and for 2 proteins (H50), respectively. Using CM10 pH4 arrays, 180 different proteins were observed. For 8 proteins, the levels were statistically different between responders and non-responders (Table III, 
Table II. Complete pathological response according to clinical variables.

\begin{tabular}{|c|c|c|c|}
\hline Characteristics & Non-response $(\mathrm{n}=114), \mathrm{n}(\%)$ & Response (n=27), n (\%) & P-value \\
\hline \multicolumn{4}{|l|}{ Surgery } \\
\hline Conservative & $68(60)$ & $21(78)$ & \multirow[t]{2}{*}{0.079} \\
\hline Mastectomy & $46(40)$ & $6(22)$ & \\
\hline \multicolumn{4}{|l|}{ Side } \\
\hline Right & $65(57)$ & $11(41)$ & \multirow{2}{*}{0.127} \\
\hline Left & $49(43)$ & $16(59)$ & \\
\hline \multicolumn{4}{|l|}{ Tumor stage } \\
\hline $\mathrm{T} 1$ & $7(6)$ & $2(7)$ & \multirow[t]{4}{*}{0.783} \\
\hline $\mathrm{T} 2$ & $66(58)$ & $17(63)$ & \\
\hline $\mathrm{T} 3$ & $24(21)$ & $6(23)$ & \\
\hline $\mathrm{T} 4$ & $17(15)$ & $2(7)$ & \\
\hline \multicolumn{4}{|c|}{ Nodal involvement } \\
\hline No & $59(52)$ & $14(56)$ & \multirow[t]{2}{*}{0.700} \\
\hline $\mathrm{N}^{+}$ & $55(48)$ & $11(44)$ & \\
\hline \multicolumn{4}{|l|}{ SBR stage } \\
\hline I & $8(9)$ & $2(10)$ & \multirow[t]{3}{*}{0.304} \\
\hline II & $63(70)$ & $10(53)$ & \\
\hline III & $19(21)$ & $7(37)$ & \\
\hline \multicolumn{4}{|l|}{ ER status } \\
\hline Negative & $36(32)$ & $18(69)$ & \multirow[t]{2}{*}{0.0004} \\
\hline Positive & $78(68)$ & $8(31)$ & \\
\hline \multicolumn{4}{|l|}{ PR status } \\
\hline Negative & $54(47)$ & $20(77)$ & \multirow[t]{2}{*}{0.0064} \\
\hline Positive & $60(53)$ & $6(23)$ & \\
\hline \multicolumn{4}{|l|}{ HER2 status } \\
\hline Negative & $70(75)$ & $16(73)$ & \multirow[t]{2}{*}{0.867} \\
\hline Positive & $24(25)$ & $6(27)$ & \\
\hline \multicolumn{4}{|l|}{ Ki-67 index } \\
\hline$<15$ & $10(24)$ & 0 & \multirow[t]{2}{*}{0.098} \\
\hline$\geq 15$ & $31(76)$ & $9(100)$ & \\
\hline
\end{tabular}

Fig. 1). The generalized ROC criterion identified cytosolic proteins $\mathrm{C} 1, \mathrm{C} 7$ and $\mathrm{C} 8$ as the best three variables. However, only $\mathrm{C} 1$ and $\mathrm{C} 7$ were selected since they were subsequently validated in 95.0 and $85.6 \%$ of models, respectively. On the other hand, C8 was only present in $59.2 \%$ of the models and was not retained. The AUC for $\log (\mathrm{C} 1)$ and $\log (\mathrm{C} 7)$ was equal to $\mathrm{AUC}=0.768(95 \% \mathrm{CI}, 0.623-0.858)($ Fig. $2 \mathrm{~A})$.

Plasma. Eighty-eight (62\%) patients were evaluable for plasma analysis. Using CM10 pH4 and H50 arrays, 85 and 107 different proteins were observed, respectively, and their levels were statistically different between responders and non-responders for 2 proteins $(\mathrm{CM} 10 \mathrm{pH} 4)$ and 6 proteins (H50). Using IMAC-Cu arrays, 98 different proteins were observed. For 12 proteins, the levels were statistically different between the two populations (Table III, Fig. 1). The generalized ROC criterion identified plasma proteins P1, P2 and P7 as the best three variables. However, only P1 and P7 were selected since they were validated in 94.8 and $97.6 \%$ of models respectively. On the other hand, P2 was only present in $55.0 \%$ of the models and was not retained. The AUC for $\log (\mathrm{P} 1)$ and $\log (\mathrm{P} 7)$ was equal to $\mathrm{AUC}=0.774(95 \% \mathrm{CI}$, 0.631-0.865) (Fig. 2B).

Cytosol and plasma combination. In an effort to use both cytosol and plasma results for constructing a combined proteomic signature, the weighted combination variable $\mathrm{Z}=2 * \mathrm{Z} \_\mathrm{C}+\mathrm{Z} \_\mathrm{P}$ was generated from the integer values of the coefficients estimated from the mROC analysis (1.088 and 0.509 , respectively). The AUC for this combination was equal to $\mathrm{AUC}=0.843$ (95\% CI, 0.692-0.933) (Fig. 2C). Overall correct classification was $87.3 \%$ (Table IV). The proteomic signature remained statistically significant when adjusted for hormone receptor status. Moreover, in the population of patients with positive $\mathrm{Ki}-67$, the proteomic signature remained statistically significant. 
Table III. pCR according to the proteomic analysis of the cytosols and the plasma.

Non-response, median (range)

Cytosols (protein molecular weight, $\mathrm{m} / \mathrm{z}$ )

C1 (3077)

C2 (4629)

C3 (44002)

C4 (116996)

C5 (3556)

C6 (3348)

C7 (5071)

C8 (5793)

Z_C $=\log (\mathrm{C} 1)+\log (\mathrm{C} 7)$

Plasma (protein molecular weight, $\mathrm{m} / \mathrm{z}$ )

P1 (7948)

P2 (4752)

P3 (74986)

P4 (89539)

P5 (116754)

P6 (115505)

P7 (101771)

P8 (8052)

P9 (7849)

P10 (7577)

P11 (100104)

P12 (43422)

$\mathrm{Z} \_\mathrm{P}=\log (\mathrm{P} 1)-2 * \log (\mathrm{P} 7)$

Cytosol and plasma

$5.5(\mathbf{1 . 1 - 8 3 . 2})$
$301.7(6.6-749.7)$
$20.6(0.1-58.1)$
$22.0(0.1-71.3)$
$6.9(0.1-133.7)$
$53.0(3.8-139.1)$
$\mathbf{2 . 9}(\mathbf{0 . 1 - 1 3 . 0})$
$0.17(0.00-0.46)$
$1.39(-0.16-2.59)$

$5.4(0.1-114.5)$

$5.0(0.1-32)$

$16.0(0.1-98.5)$

$33.9(9.9-172.7)$

$14.0(1.5-59.7)$

$2.8(0.4-13.8)$

$4.0(0.2-11.0)$

$5.4(2.6-9.4)$

$1.7(0.7-3.9)$

$1.6(0.1-4.7)$

$0.9(0.1-3.7)$

$0.8(0.1-2.9)$

$-0.41(-2.49-1.67)$
Response,

median (range)

P-value

re

3.1 (0.1-23.0)

$164.7(6.8-549.5)$

$<0.0001$

$14.0(1.8-40.9)$

0.035

$13.1(2.0-35.7)$

0.024

$3.1(0.6-51.8)$

0.0116

25.9 (2.8-149.9)

0.009

2.0 (0.6-3.9)

0.056

0.006

$0.16(0.05-0.23)$

0.022

$0.77(-1.11-1.57)$

$<0.00001$

$2.9(0.1-19.9)$

0.022

$2.1(0.1-11.1)$

0.036

$8.4(0.1-31.5)$

0.0398

$17.7(8.8-132.2)$

0.012

$8.3(3.6-53.3)$

0.026

$4.1(1.1-14.9)$

0.092

$5.5(0.9-8.8)$

$\mathbf{0 . 0 2 9}$

$6.4(3.7-10.3)$

0.021

$2.3(0.9-4.7)$

0.080

$2.4(0.8-4.8)$

0.048

$1.4(0.2-3.3)$

0.039

$1.3(0.3-3.1)$

0.035

$-0.90(-2.85--0.29)$

0.0004

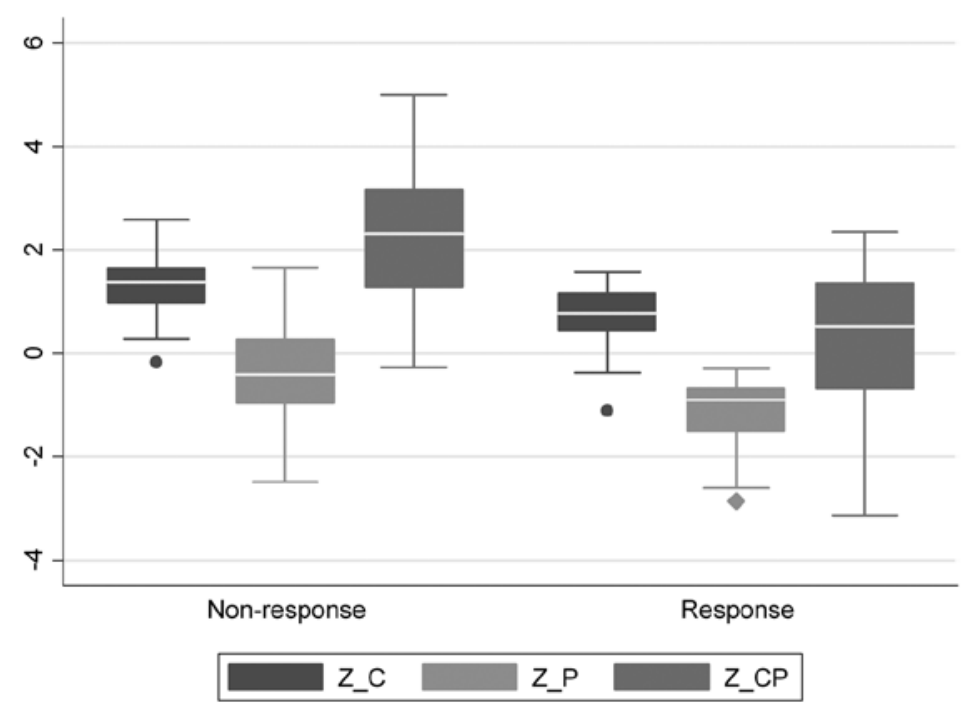

Figure 1. Box plot of $Z_{-} C, Z_{-} P$ and the combination $Z_{-} C P$ according to the response.

Due to missing data, only 63 (45\%) samples could be evaluated for the combination. However, no significant difference in patient and tumor characteristics were noted for patients included and excluded from the combination analysis (data not shown). 
A

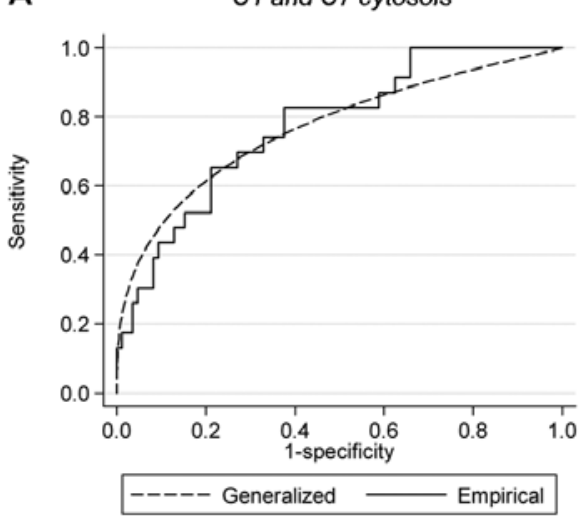

B

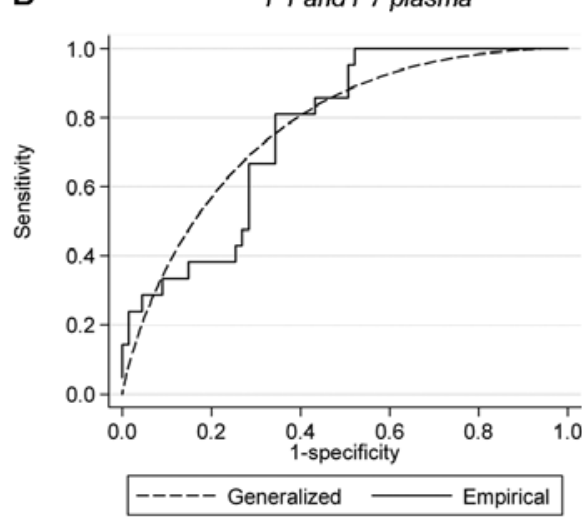

C

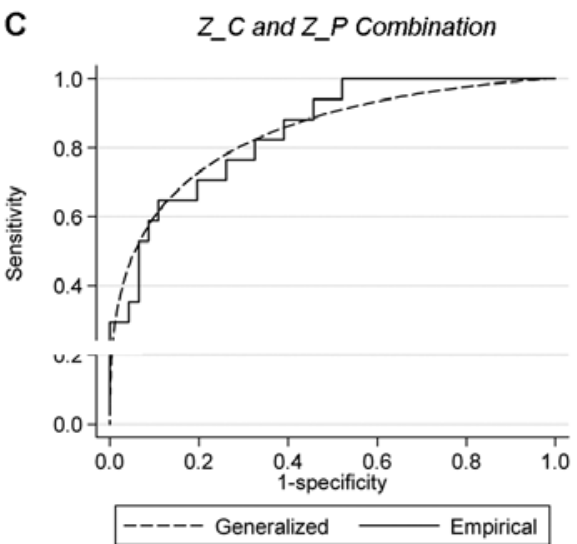

Figure 2. ROC curves for cytosols, plasma and the combination.

Table IV. ROC analysis of the cytosols and plasma combination.

\begin{tabular}{lc}
\hline Term & Result \\
\hline AUC (95\% CI) & $0.84(0.69-0.93)$ \\
Sensitivity (\%) & 89.1 \\
Specificity (\%) & 82.4 \\
Positive predictive value (\%) & 93.2 \\
Negative predictive value (\%) & 73.7 \\
Overall correct classification (\%) & 87.3 \\
\hline
\end{tabular}

\section{Discussion}

Our results showed that proteomic analysis in the plasma, in the tumor and a combination of results in the plasma and the tumor could differentiate complete pathological responders and non-responders in breast cancer patients receiving neoadjuvant chemotherapy. Two plasma and two cytosolic proteins were selected and a combined plasma and tumor signature was generated. This signature remained statistically significant when adjusted for hormone receptor status and in Ki-67positive patients.

Overall, the study population was similar to the patient population of other neoadjuvant studies. Ninety-four percent of patients had a tumor $>2 \mathrm{~cm}$ (median tumor size, $4 \mathrm{~cm}$ ). Nearly half of them had positive axillary nodes. Only $61 \%$ of the tumors had positive estradiol receptors and $47 \%$ were positive for progesterone receptors. Twenty-four percent of tumors were grade 3 and the median value of $\mathrm{Ki}-67$ was $30 \%$. Twenty-five percent of tumors were HER2-positive (either by immunohistochemistry or SISH). This population had a high tumor burden and aggressive tumor.

SELDI-TOF MS profiling appears to be a promising tool for diagnosis $(6-7,11,19)$. The use of such a technique in relation to therapeutic response prediction has been limited (20). In the present study, we used this technology on both plasma and cytosols to predict the therapeutic response in a series of breast cancer patients receiving neoadjuvant chemotherapy.
In both plasma and cytosols, the assays were performed using three types of ProteinChip arrays (CM10 pH4, IMAC-Cu and H50). In cytosols, CM10 pH4 ProteinChip arrays appeared to be the best arrays to discriminate between responder and non-responder patients, whereas IMAC-Cu array was the best discriminator in plasma.

In the present study, no data was contained concerning the characterization of the proteins of the established signature. It is noteworthy that relevant biomarkers identified in SELDI-TOF profiling studies are in most cases non-specific host response-generated proteins at rather high levels (21). For example, in such studies, haptoglobin, transferrin, or the C3A or C3B complement have been evidenced. Furthermore, ubiquitin and ferritin light chain, corresponding to the two peaks of interest, were evidenced using SELDI-TOF-MS screening to identify differentially expressed cytosolic proteins with a prognostic impact in node-negative breast cancer patients with no relapse vs. patients with metastatic relapse (22).

We demonstrated that plasma and tumor proteomic profiles obtained prior to neoadjuvant chemotherapy in primary breast cancer may predict a complete pathological response to treatment. Comparable results were found by $\mathrm{He}$ et al (15) in a smaller population. In the entire group, a single peak at mass/ charge ratio $(\mathrm{m} / \mathrm{z})$ of 16,906 correctly separated $88.9 \%$ of the tumors with pathological complete response and $91.7 \%$ of the resistant tumors. These data suggest that breast cancer protein biomarkers may be used to pre-select patients for optimal chemotherapeutic treatments. Other studies have identified potential markers related to neoadjuvant chemotherapy using SELDI-TOF MS by comparing the proteomic profiles before and after treatment. Relatively few changes were identified in plasma by Pusztai et al (23). They detected only a single chemotherapy-inducible SELDI-MS peak [mass/charge ratio $(\mathrm{m} / \mathrm{z}), 2790]$ that was induced by paclitaxel and, to a lesser extent, by FAC chemotherapy. Recently, the intensities of eight different protein peaks were demonstrated to be higher in breast cancer tissue extracts after neoadjuvant chemotherapy than those before neoadjuvant chemotherapy (24). Although further experiments are needed to prove the reliability of these eight proteins, these results will help in the establishment of protein models based on drug resistance-related protein peaks 
to screen whether a patient is suitable for adopting neoadjuvant chemotherapy and to improve cancer treatment.

The pCR according to Sataloff (TA NA, TA, NB) was in the lower range of the published results for anthracycline-taxane combining regimens (25). One reason for this relatively low rate could be the very restrictive definition of the $\mathrm{pCR}$ related to the surgical specimen process for pathological analysis as previously described (26). The prognostic factors of response were $\mathrm{ER}(\mathrm{P}=0.0004)$ and $\mathrm{PR}(\mathrm{P}=0.006)$ negativity and Ki-67 above the median value of $30 \%(\mathrm{P}=0.003)$. No pCR was observed for Ki-67 below 15\%. These factors have been previously reported in many neoadjuvant studies (27). Similarly, the pCR rate was higher in triple-negative tumors (28) and in ductular than in lobular cancer (29). Several molecular signatures are being tested in the neoadjuvant setting (30-33). However, the benefit of using genetic predictors over usual pathological biomarkers is not clear. Our study showed that a proteomic analysis of plasma and cytosol could also predict pCR in breast cancer patients treated with FEC and docetaxel. The role of a proteomic analysis in clinical practice remains to be defined.

\section{References}

1. Greenlee RT, Murray T, Bolden S and Wingo PA: Cancer statistics, 2000. CA Cancer J Clin 50: 7-33, 2000.

2. Goldhirsch A, Wood WC, Gelber RD, Coates AS, Thurlimann B and Senn HJ: Meeting highlights: updated international expert consensus on the primary therapy of early breast cancer. J Clin Oncol 21: 3357-3365, 2003.

3. Roche H, Fumoleau P, Spielman M, et al: Sequential adjuvant epirubicin-based and docetaxel chemotherapy for node-positive breast cancer patients: the FNCLCC PACS 01 Trial. J Clin Oncol 24: 5664-5671, 2006.

4. Vercoutter-Edouart AS, Lemoine J, Le Bourhis X, et al: Proteomic analysis reveals that 14-3-3sigma is down-regulated in human breast cancer cells. Cancer Res 61: 76-80, 2001.

5. Adam BL, Qu Y, Davis JW, et al: Serum protein fingerprinting coupled with a pattern-matching algorithm distinguishes prostate cancer from benign prostate hy perplasia and healthy men. Cancer Res 62: 3609-3614, 2002.

6. Petricoin EF, Ardekani AM, Hitt BA, et al: Use of proteomic patterns in serum to identify ovarian cancer. Lancet 359: 572-577, 2002

7. Petricoin EF III, Ornstein DK, Paweletz CP, et al: Serum proteomic patterns for detection of prostate cancer. J Natl Cancer Inst 94: 1576-1578, 2002

8. Vlahou A, Laronga C, Wilson L, et al: A novel approach toward development of a rapid blood test for breast cancer. Clin Breast Cancer 4: 203-209, 2003.

9. Koopmann J, Zhang Z, White N, et al: Serum diagnosis of pancreatic adenocarcinoma using surface-enhanced laser desorption and ionization mass spectrometry. Clin Cancer Res 10: 860-868, 2004.

10. Wadsworth JT, Somers KD, Cazares LH, et al: Serum protein profiles to identify head and neck cancer. Clin Cancer Res 10: 1625-1632, 2004.

11. Zhang Z, Bast RC Jr, Yu Y, et al: Three biomarkers identified from serum proteomic analysis for the detection of early stage ovarian cancer. Cancer Res 64: 5882-5890, 2004.

12. Fung ET, Yip TT, Lomas L, et al: Classification of cancer types by measuring variants of host response proteins using SELDI serum assays. Int J Cancer 115: 783-789, 2005.

13. Fung ET, Thulasiraman V, Weinberger SR and Dalmasso EA: Protein biochips for differential profiling. Curr Opin Biotechnol 12: $65-69,2001$

14. Hodgkinson VC, Eagle GL, Drew PJ, Lind MJ and Cawkwell L: Biomarkers of chemotherapy resistance in breast cancer identified by proteomics: current status. Cancer Lett 294: 13-24, 2010 .
15. He J, Shen D, Chung DU, Saxton RE, Whitelegge JP, Faull KF and Chang HR: Tumor proteomic profiling predicts the susceptibility of breast cancer to chemotherapy. Int J Oncol 35: 683-692, 2009.

16. Sataloff DM, Mason BA, Prestipino AJ, Seinige VL, Lieber CP and Baloch Z: Pathologic response to induction chemotherapy in locally advanced carcinoma of the breast: a determinant of outcome. J Am Coll Surg 180: 297-306, 1995.

17. Bradford MM: Rapid and sensitive method for the quantitation of microgram quantities of protein utilizing the principle of protein-dye binding. Anal Biochem 72: 248-254, 1976.

18. Kramar A, Farraggi D, Fortuné A and Reiser B: mROC: a computer program for combining tumour markers in predicting disease states. Comput Methods Programs Biomed 66: 199-207, 2001.

19. Kozak KR, Amneus MW, Pusey SM, et al: Identification of biomarkers for ovarian cancer using strong anion-exchange ProteinChips: potential use in diagnosis and prognosis. Proc Natl Acad Sci USA 100: 12343-12348, 2003.

20. Gonçalves A, Esterni B, Bertucci F, et al: Postoperative serum proteomic profiles may predict metastatic relapse in high-riskprimary breast cancer patients receiving adjuvant chemotherapy. Oncogene 25: 981-989, 2006.

21. Diamandis EP: Analysis of serum proteomic patterns for early cancer diagnosis: drawing attention to potential problems. J Natl Cancer Inst 96: 353-356, 2004.

22. Ricolleau G, Charbonnel C, Lodé L, et al: Surface-enhanced laser desorption/ionization time of flight mass spectrometry protein profiling identifies ubiquitin and ferritin light chain as prognostic biomarkers in node-negative breast cancer tumors. Proteomics 6: 1963-1975, 2006.

23. Pusztai L, Gregory BW, Baggerly KA, et al: Pharmacoproteomic analysis of prechemotherapy and postchemotherapy plasma samples from patients receiving neoadjuvant or adjuvant chemotherapy for breast carcinoma. Cancer 100: 1814-1822, 2004.

24. Zhang K, Yuan K, Wu H, et al: Identification of potential markers related to neoadjuvant chemotherapy sensitivity of breast cancer by SELDI-TOF MS. Appl Biochem Biotechnol 166: 753-763, 2012.

25. Dang CT and Hudis C: Preoperative chemotherapy for operable breast cancer. In Diseases of the Breast. Harris JR, Lippman ME, Morrow M and Osborne CK (eds). WoltersKluwer/ Lippincott Williams \&Williams, Philadelphia, pp715-723, 2010.

26. Mailliez A, Baranzelli MC, Giard S, et al: Is there a reliable method to assess the complete pathologic response on the tumor after neo-adjuvant chemotherapy in inflammatory breast cancer toward recommendations for the pathologic process? Experience in 56 patients treated in a single institution. Breast J 16: 464-471, 2010.

27. Penault-Llorca F, Abrial C, Raolelfils I, et al: Changes and predictive and prognostic value of the mitotic index, Ki-67, cyclin D1, and cyclo-oxygenase-2 in 710 operable breast cancer patients treated with neoadjuvant chemotherapy. Oncologist 13: 1235-1245, 2008

28. Liedtke C, Mazouni C, Hess KR, et al: Response to neoadjuvant therapy and long-term survival in patients with triple-negative breast cancer. J Clin Oncol 26: 1275-1281, 2008.

29. Katz A, Saad ED, Porter P and Pusztai L: Primary systemic chemotherapy of invasive lobular carcinoma of the breast. Lancet Oncol 8: 55-62, 2007.

30. Liedtke C, Hatzis C, Symmans WF, et al: Genomic grade index is associated with response to chemotherapy in patients with breast cancer. J Clin Oncol 27: 3185-3191, 2009.

31. Straver ME, Glas AM, Hannemann J, et al: The 70-gene signature as a response predictor for neoadjuvant chemotherapy in breast cancer. Breast Cancer Res Treat 119: 551-558, 2010.

32. Tabchy A, Valero V, Vidaurre T, et al: Evaluation of a 30-gene paclitaxel, fluorouracil, doxorubicin, and cyclophosphamide chemotherapy response predictor in a multicenter randomized trial in breast cancer. Clin Cancer Res 16: 5351-5361, 2010.

33. Lee JK, Coutant C, Kim YC, et al: Prospective comparison of clinical and genomic multivariate predictors of response to neoadjuvant chemotherapy in breast cancer. Clin Cancer Res 16: 711-718, 2010. 